\title{
The significance of variations in immunoreactive and clottable fibrinogen in health and following thrombosis
}

\author{
P. WOLF 1 , G. W. FARRELL, AND K. W. WALTON \\ From the Department of Experimental Pathology, University of Birmingham
}

SYNOPSIS In individuals in a steady state of fibrinogen metabolism, immunoreactive and clottable fibrinogen estimates of plasma fibrinogen show close agreement. These estimates also detect any increase of plasma fibrinogen (due to increased synthesis) following metabolic stresses but in certain circumstances discrepancies between immunoreactive and clottable fibrinogen values occur which are of diagnostic assistance.

During extensive thrombosis, circulating 'cryoprofibrin' (fibrin intermediates) may be formed. These fail to give full quantitative immunodiffusion reactions with antifibrinogen. Values for immunoreactive are therefore lower than for clottable fibrinogen.

When intravascular catabolism (due to plasmin action) accompanies increased synthesis, since some of the molecular breakdown products of fibrin or fibrinogen react with antifibrinogen but are not clottable, immunoreactive values exceed those for clottable fibrinogen. This discrepancy is therefore an indicator of thrombolysis.

Each discrepancy in turn may be encountered during the alternating predominance of thrombosis or thrombolysis in vivo: (a) physiologically, in association with the menstrual cycle; (b) pathologically, following surgical operations or extensive intravascular thrombosis.

Fibrinogen is a rapidly metabolized protein with a relatively short biological half-life of about three to six days (MacFarlane, Todd, and Cromwell, 1964; Takeda, 1966). Like other 'acute phase reactant' proteins, fibrinogen is synthesized rapidly in response to trauma, burns, acute infections, and conditions associated with haemorrhage and tissue breakdown. As a consequence, the plasma concentration shows a rapid rise. From the results of metabolic studies it has been suggested that the stimulus to increased synthesis of fibrinogen may be increased catabolism of the molecule (Reeve, Takeda, and Atencio, 1966). Depending upon the clinical circumstances, different enzymes may be involved in the intravascular breakdown of fibrinogen either before, during, or after its conversion to fibrin.

For example, during blood coagulation, thrombin produces a specific partial proteolysis of the fibrinogen molecule resulting in the splitting off of two pairs

${ }^{1}$ Member of external scientific research staff, Medical Research Council, on attachment to the Department of Experimental Pathology, University of Birmingham.

Received for publication 18 July 1971 of peptide units (Bailey and Bettelheim, 1955) now known as fibrinopeptides A and B (Blömback, 1967). Following their detachment, the residual portions of the molecule polymerize to form insoluble fibrin (Fig. 1A). Fibrinopeptides A and B evidently do not contribute substantially to the antigenicity of the original molecule since antisera directed against fibrin are indistinguishable from antisera to fibrinogen.

On the other hand, when either fibrin or fibrinogen is exposed to plasmin action (Fig. 1B) proteolysis is more extensive and a large number and variety of peptide fragments is released. The end products of complete lysis of fibrinogen, although incoagulable by thrombin (Lipinski, Wegrzynowicz, Budzynski, Kopeć, Latallo, and Kowalski, 1967), react with antifibrinogen antibody. Certain of the fragments give precipitin lines in agar diffusion reactions (the $D$ and $E$ fragments of Nussenzweig, Seligman, and Grabar, 1967) while others behave as non-precipitating antigens. Some of the breakdown products of fibrin are similarly identifiable by immunological methods. 
Antigen Enzyme Reaction of digestion product with antibody

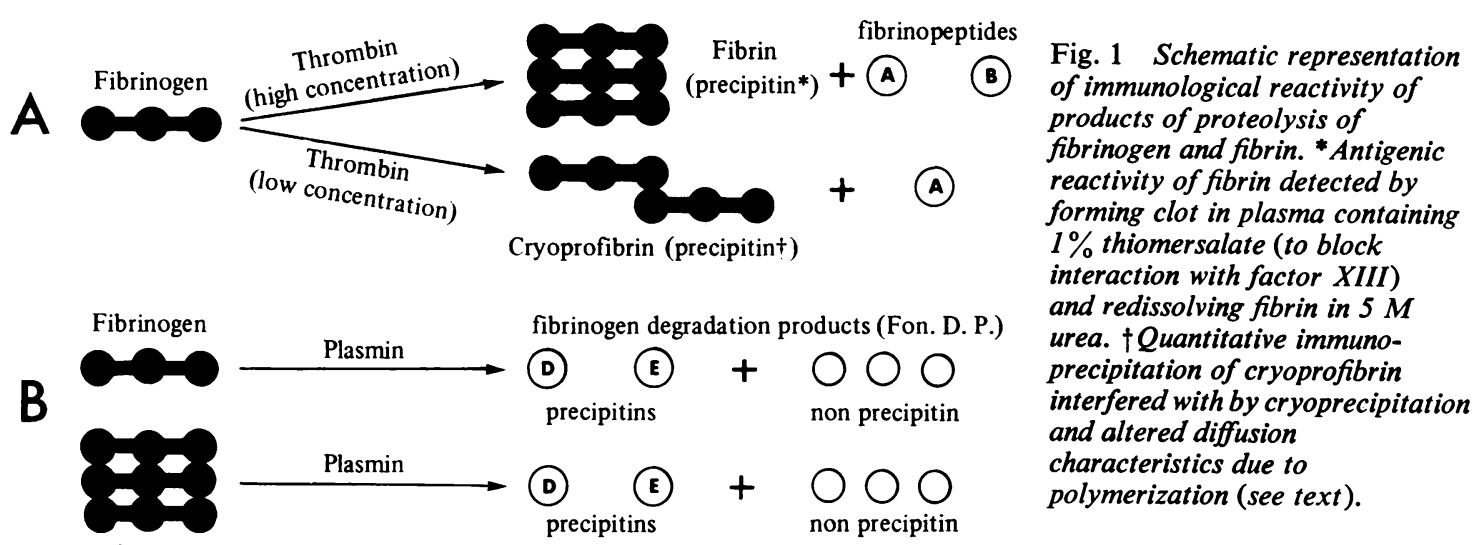

Fibrin

fibrin degradation products (F. D. P.)

The detection of these fragments in serum has been advocated as a test for the occurrence of fibrinolysis (or fibrinogenolysis) in various clinical conditions (Merskey, Kleiner, and Johnson, 1966). However, this test, in itself, does not establish whether the fragments were circulating in the plasma or were subsequently formed in vitro as a result of accelerated clot lysis in the test tube.

As an alternative approach, it has been found that parallel quantitative comparisons of immunoreactive and clottable fibrinogen provide new information about the biological behaviour of fibrinogen and of these degradation products, while discrepancies between these two kinds of assay may be of practical significance in the assessment of thrombosis and thrombolysis in clinical practice, when these processes are substantial in degree but difficult otherwise to detect and appraise.

\section{Materials and Methods}

DETERMINATION OF CLOTTABLE FIBRINOGEN This was carried out by a colorimetric modification of the method of Fearnley and Chakrabarti (1966) as described by Farrell and Wolf (1971).

\section{DETERMINATION OF IMMUNOREACTIVE FIBRINOGEN}

In normal plasma this was performed by the methods of Wolf and Walton (1965). For measurement of fibrinogen suspected to be in abnormal polymerized form this method was modified as follows: the sodium chloride concentration in the agar and diluting fluid was increased from $0.15 \mathrm{M}$ to $3 \mathrm{M}(17 \%$ sodium chloride) but the concentration of dipotassium 3* sequestrene and the $p \mathrm{H}$ of these media were unaltered.

\section{PREPARATION OF ANTIHUMAN FIBRINOGEN ANTISERUM}

This was carried out by immunizing rabbits as described by Farrell and Wolf (1970).

\section{MEASUREMENT OF DIFFUSION}

CHARACTERISTICS OF FIBRINOGEN

A modification of the method of Allison and Humphrey (1960) as applied to measuring the diffusion of human plasminogen (Farrell, Stuckey, and Wolf, 1969) was used, substituting rabbit antifibrin for rabbit antiplasmin.

\section{HUMAN THROMBIN}

Human thrombin was prepared by the method of Biggs and Macfarlane (1957). The thrombin solution was freeze-dried in $\mathbf{0 . 2} \mathrm{ml}$ aliquots and reconstituted before use to give a solution which clotted an equal volume of fresh citrated plasma in 10 to 13 seconds.

\section{STREPTOKINASE}

This was obtained from Kabi Biochemicals Ltd. Dried streptokinase (marked 'for laboratory use only') was reconstituted with distilled water to give a concentration of 200 units $/ \mathrm{ml}$. The solutions were stored in $0.5 \mathrm{ml}$ quantities at $-30^{\circ}$ until required. The solutions were thawed, used immediately, and any unused residue was discarded.

\section{DIGESTION OF FRESH PLASMA CLOTS}

To $0.3 \mathrm{ml}$ plasma, in a water bath at $37^{\circ}$, was added $0.1 \mathrm{ml}$ of $0.15 \mathrm{M}$ sodium chloride solution and 
$0.1 \mathrm{ml}$ of streptokinase solution (containing 200 units $/ \mathrm{ml}$ ). After exactly one minute $0.1 \mathrm{ml}$ of thrombin solution was added. A clot formed which dissolved within 60 to 120 seconds. The digest was incubated for a further 10 minutes before serial dilutions were made for immunological determinations.

\section{BLOOD COLLECTION}

Blood was collected in trisodium citrate or dipotassium sequestrene as detailed elsewhere (Farrell and Wolf, 1971). In some instances (see text) epsilon amino caproic acid (EACA) was also included to give a final concentration of $2.6 \mathrm{~g} / 100 \mathrm{ml}(0 \cdot 2 \mathrm{M})$.

\section{DETECTION OF FIBRIN OR FIBRINOGEN}

DEGRADATION PRODUCTS

This was carried out by immunodiffusion and by immunoelectrophoresis of serum, collected with and without the addition of EACA at the concentration detailed above, using antifibrinogen antiserum.
Results

IMMUNOREACTIVE AND CLOTTABLE

FIBRINOGEN IN HEALTHY SUBJECTS

When immunoreactive fibrinogen levels, as determined by the method of Wolf and Walton (1965), were compared with clottable fibrinogen levels, values lying between 250 and $450 \mathrm{mg} / 100 \mathrm{ml}$ were obtained in 23 healthy ambulant subjects $(11$ males and 12 females). In all but two subjects (both female and referred to in more detail below) the concordance between the two methods was acceptable when allowance was made for the $2+1$ dilution system adopted for the immunological estimate (Fig. 2).

In two females yielding discrepant results (see points indicated by arrows in Fig. 2) the immunological estimate exceeded the clottable value by a wide margin. These results were associated with other findings suggesting that these subjects differed from the remainder in showing evidence of proteolysis of fibrinogen both in vitro and in vivo. For example, in

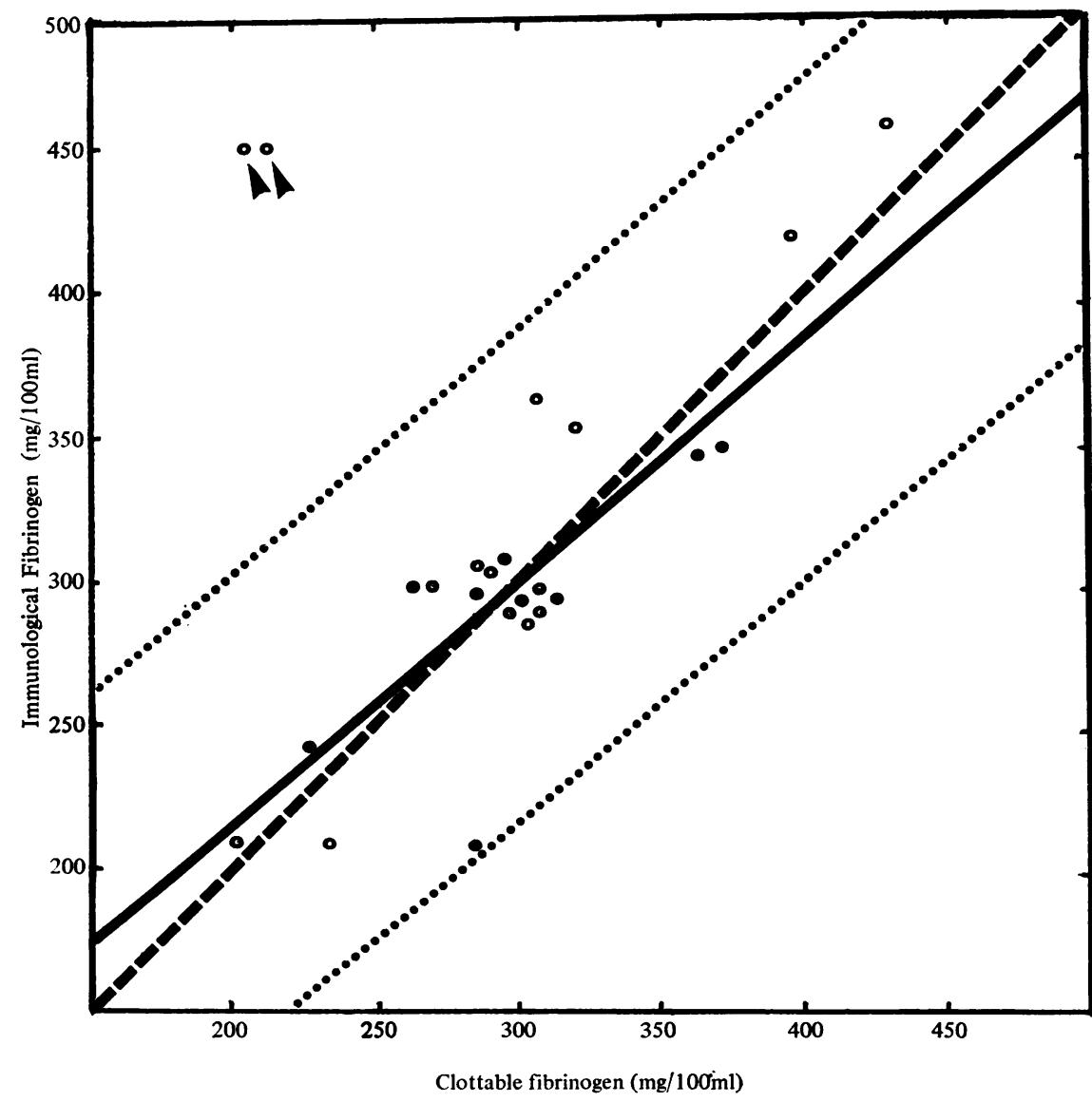

Fig. 2 Comparison of measurements of immunoreactive and clottable fibrinogen in 23 healthy ambulant subjects (closed symbols males; open symbols females). Regression (continuous line) and 95\% confidence limits (dotted lines) compared with theoretical slope (dashed line). Two results marked by arrows in top left-hand corner discarded in calculating correlation coefficient, $r: 0.752$, $P>0.001$ (see text). 
both subjects (though not in the remainder) 'fibrin fragments' were demonstrated immunologically in the serum of blood collected in EACA to prevent subsequent fibrinolysis (see Methods) suggesting the presence of circulating fibrin or fibrinogen degradation products. In addition both subjects showed progressive decrease of fibrin yield from citrated blood (collected without EACA present) after incubation of the blood at $37^{\circ}$ for one and two hours, demonstrating accelerated clot lysis in the test tube.

When the results from these two subjects were discarded, a highly significant correlation coefficient $(r: 0.752 ; \mathrm{P}>0.001)$ was obtained with a slope not significantly different from the theoretical one (Fig. 2). Statistical comparison of means by Student's $t$ test showed no significant difference between the estimates by the two techniques for either males or females (Table). Similarly, no significant difference was found between the overall mean values between the sexes although the standard deviation was larger for females. This is also seen from Fig. 2 where a wider scatter of individual values for females is evident.

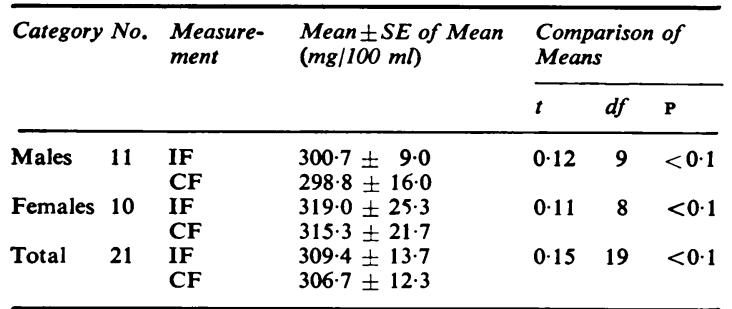

Table Immunoreactive (IF) and clottable fibrinogen $(C F)$ in healthy subjects ${ }^{1}$

${ }^{1}$ Overall means + SE: males $299 \cdot 8 \pm 10 \cdot 8$; females $317.6 \pm 10 \cdot 7$; total: $308 \cdot 0 \pm 10 \cdot 7$

The occurrence of markedly discrepant results in two females and the general tendency for results in females to show a wider scatter suggested a greater lability of plasma fibrinogen concentration in women which might be physiological in origin. Sequential examination of immunoreactive and clottable fibrinogen levels in the two subjects showing the discrepant results and in three additional women was undertaken at various times in relation to the menstrual cycle to investigate this possibility. Similar discrepancies between immunoreactive and clottable fibrinogen levels were again observed in the two subjects in whom these were originally noted. But the discrepancy varied in its magnitude, being maximal in the premenstrual phase when clottable fibrinogen values were often minimal while immunoreactive fibrinogen values rose to a peak (Fig. 3).
Similar fluctuations in immunoreactive and clottable fibrinogen levels were observed in the three other female subjects tested but were variable in degree between different women and even in the same woman between different cycles. Values for immunoreactive tended to exceed those for clottable fibrinogen, particularly markedly when a minor infective process (eg, a tooth abscess, as shown in Fig. 3) coincided with the premenstrual phase of the cycle. On the other hand, little or no fluctuation in immunoreactive or clottable fibrinogen levels was observed by sequential tests in healthy males except where tests were carried out during coincident minor infections (Fig. 3) when immunoreactive levels sometimes showed a tendency to exceed clottable fibrinogen values.

The most marked elevation of immunoreactive values above clottable fibrinogen values coincided with the appearance of 'fibrin fragments' in the serum in the premenstrual phase. It was concluded that the discrepancy between immunoreactive and clottable fibrinogen values probably therefore arose because the immunological method detected both the increased level of circulating fibrinogen and also circulating 'fibrin fragments' generated by the breakdown of endometrium, haemorrhage, and clot lysis which must occur in this phase of the cycle. On the other hand, the 'fibrin fragments', being incoagulable by thrombin, would not similarly contribute to the clottable fibrinogen value except when actually incorporated into the clot. This was found sometimes to be the case (see below).

\section{IMMUNOREACTIVE AND CLOTTABLE}

FIBRINOGEN AFTER MAJOR SURGERY

Surgical operations are necessarily attended by localized thrombosis in and around the operation area. During repair and regeneration there is lysis of both intra- and extravascular fibrin deposits. Since the aim was to examine the relation between thrombosis and thrombolysis, a preliminary study in surgical patients offered several advantages. These were that measurements could be made both before and after elective operations, each patient thus serving as his own control; the time at which thrombosis commenced could be gauged with reasonable accuracy; and some estimate of the probable extent of thrombosis could be obtained from knowledge of the nature and extent of the operative procedure.

When immunoreactive and clottable fibrinogen levels were examined in such patients, changes similar in kind to those already described in some women during the menstrual cycle were observed consistently during the postoperative period (from about the second or third day after operation). The 


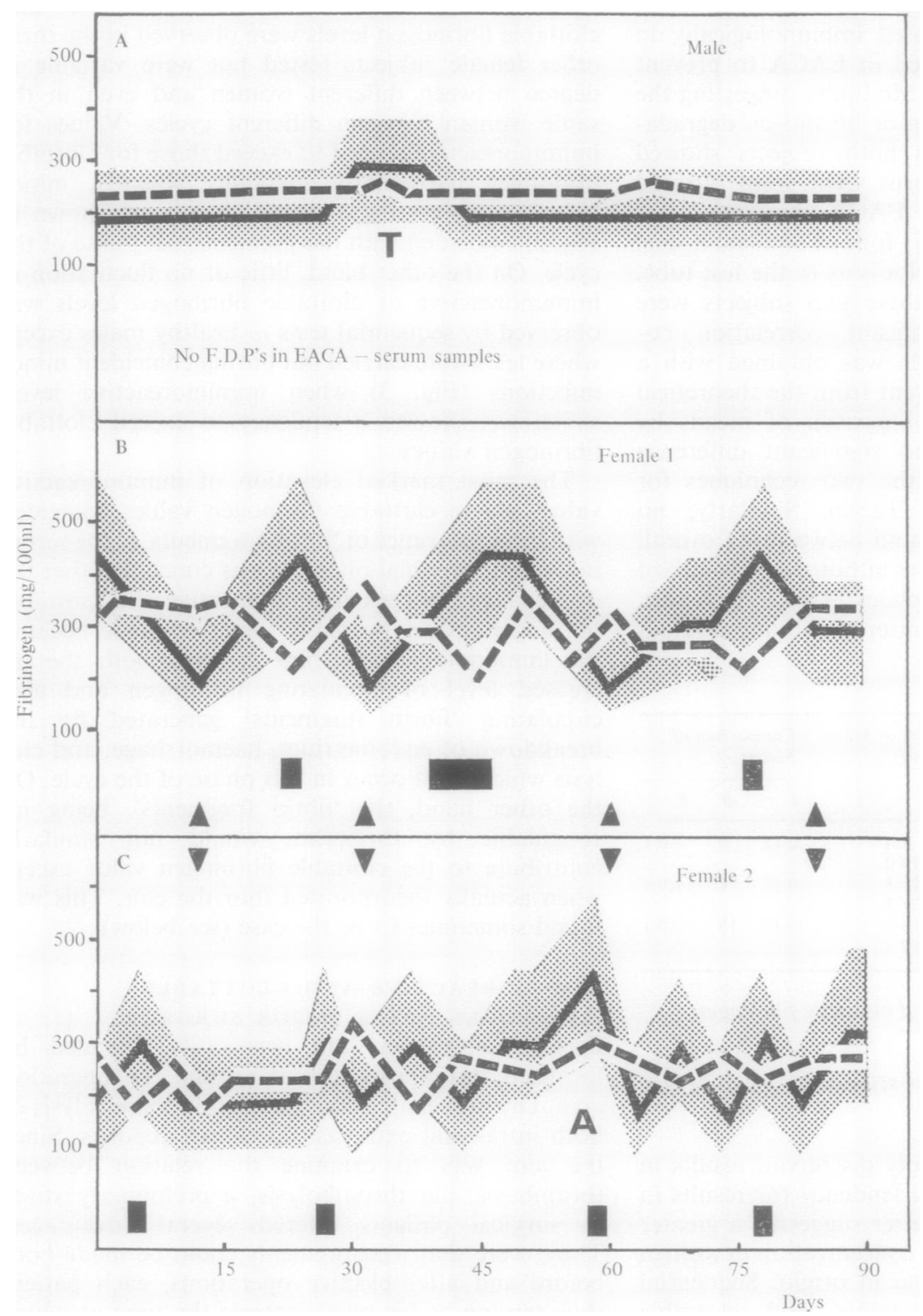

Fig. 3. Sequential comparisons of immunoreactive fibrinogen (IF) plotted as continuous line and confidence limits (shaded area) with clottable fibrinogen $(C F)$ plotted as dashed line, in a healthy male (A) and in two females (B and C). The results in $\mathrm{B}$ and $\mathrm{C}$ relate to the same subjects giving the abnormal values indicated in Fig. 1 when a single casual estimate was made. Note constancy and agreement of estimates in the male and effect on values of minor throat infection (T) in Fig $3 a$. In contrast note lability of both immunoreactive and clottable fibrinogen in females and extent of discrepancies between estimates in premenstrual phases of cycle (menstrual periods indicated by triangles). Solid blocks indicate appearance of FDP in serum. Also note additional effects of dental abscess (A) in Fig. 3C. 


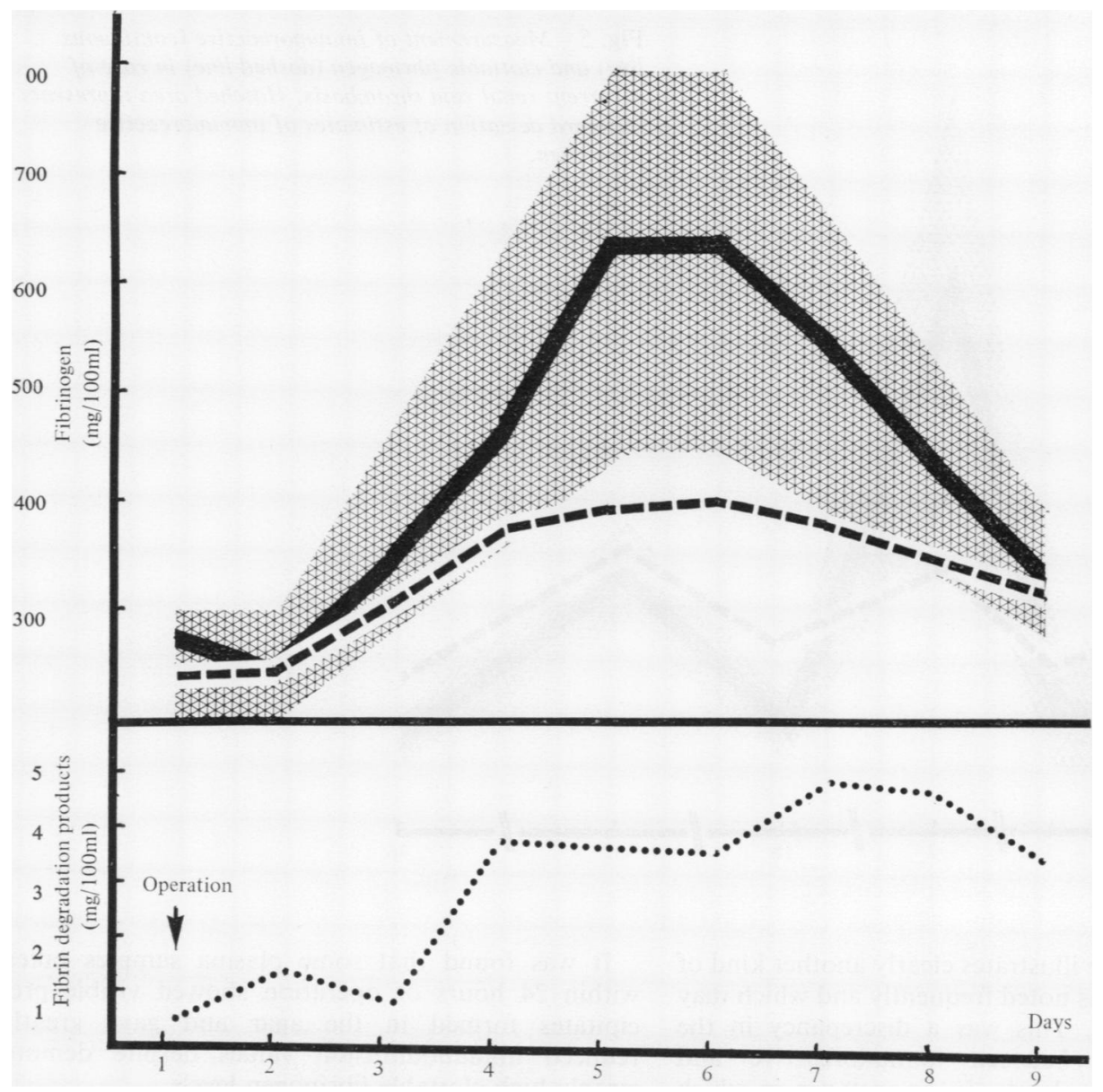

Fig. 4 Daily comparison of immunoreactive fibrinogen (continuous line with hatched area representing confidence limits) and clottable fibrinogen (dashed line) in man of 46 before and after removal of benign pleural tumour. Operation at point marked by arrow. Note that maximal discrepancy between immunoreactive and clottable fibrinogen doesnot correspond with appearance of fibrin degradation products in serum (see text).

changes were more marked following surgery, however, and lasted for seven to ten days, depending upon the severity of the operation. This sequence is shown for a typical case (daily estimations before and after removal of a benign pleural tumour in a man of 46 years) in Figure 4 . It can be seen that both immunoreactive and clottable fibrinogen values rose sharply, reaching a peak between days 5 and 6 after operation and then declining. Over the period of formation of this peak, immunoreactive values considerably exceeded clottable fibrinogen values while fibrin degradation products in the serum showed a similar rise and fall but one not precisely related to that of immunoreactive values (see below).

Two episodes of fluctuation of fibrinogen levels, sequentially followed, were seen in another patient, illustrated in Figure 5. In this case renal vein thrombosis had been established radiographically (clinical details to be reported separately by Hardwicke $e t a l$ ) before fibrinogen levels were measured.
By the seventh day after catheterization of the renal vein there was a sharp increase in immunoreactive and clottable fibrinogen levels, the former very greatly exceeding the latter. During this period of increase of fibrinogen levels, there was an accompanying increase of fibrin degradation products in the serum. However, in this as in the previous case, the period of appearance of these fragments was not closely related to the period of elevation of immunoreactive and clottable fibrinogen values. This was thought to be because simultaneously demonstrable accelerated fibrinolysis in vitro contributed to the fragments found.

In the late postoperative period a further extension of renal vein thrombosis was followed by another sharp rise in fibrinogen levels. But on this occasion there was no appreciable discrepancy between immunoreactive and clottable fibrinogen levels at the peak of response and no increase of fibrin fragments was noted in serum. 


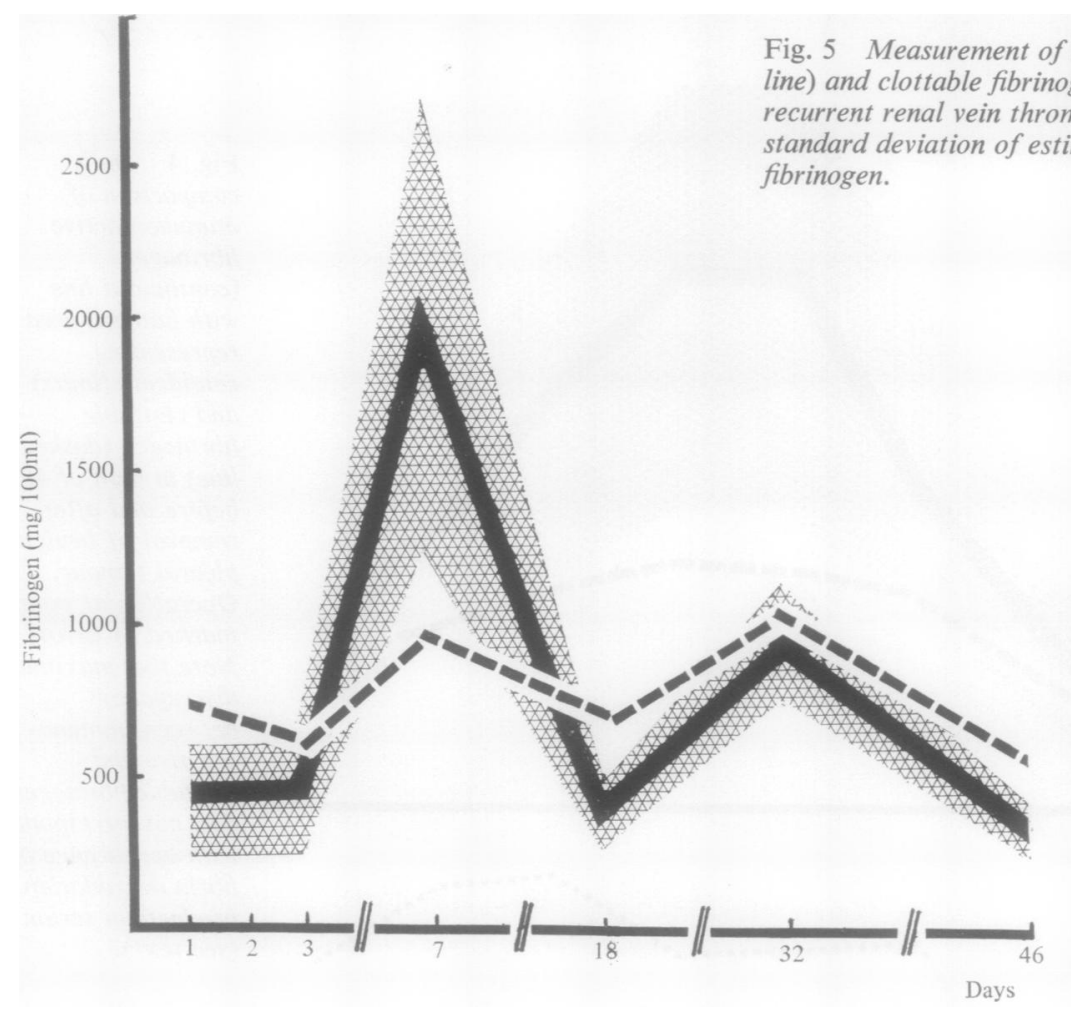

This second case illustrates clearly another kind of anomaly which was noted frequently and which may be of significance. This was a discrepancy in the reverse direction between immunoreactive and clottable fibrinogen levels, ie, an instance in which immunoreactive was found to be markedly below clottable fibrinogen level. This is seen to be the case in this patient before operation (when thrombosis of the renal vein was known to be in progress), for about 36 hours after operation, and again on the 18 th day when recurrence of renal vein thrombosis was detected.

In other previously healthy individuals subjected to elective surgery, immunoreactive and clottable fibrinogen levels showed the expected agreement before and immediately after surgery. But within the first 24-48 hours of the postoperative period a transient drop of immunoreactive below clottable fibrinogen levels often occurred. Plasma taken at this time almost always showed the formation of cryoprecipitates within four hours of storage at $4^{\circ}$. The following observations and experiments showed these to be sparingly soluble fibrinogen aggregates which were coagulable by thrombin but which behaved anomalously in immunodiffusion to give rise to underestimates of immunoreactive fibrinogen.
It was found that some plasma samples taken within 24 hours of operation showed visible precipitates formed in the agar and gave greatly reduced immunodiffusion values despite demonstrably high clottable fibrinogen levels.

Using the technique of Allison and Humphrey (1960) in those instances in which the abnormal plasmas did not show precipitate formation in the agar, it was possible to show that although material reacting with antifibrinogen was present, it showed abnormal diffusion characteristics compatible with an increase of molecular size (presumably polymerization).

When normal plasma or normal fibrinogen was added to these abnormal plasmas, even the additionally added fibrinogen could not be accurately measured by immunodiffusion. But clottable fibrinogen values in such cases showed the expected increment.

Since the fibrinogen molecule in these plasmas seemed altered in physical form so as to be less immunoreactive but still clottable, it seemed of interest to examine the effect of its complete conversion to fibrin and subsequent digestion. Fibrin clots of this kind were digested by streptokinaseinduced plasmin action (see Methods) and the yield 
of digestion products was compared with that of normal plasmas with equal clottable fibrinogen values. In many cases the abnormal plasma samples yielded peptides with similar diffusion properties and at the same concentration as the matched normal plasmas. But in some instances, the pathological plasma samples yielded digests which still gave immunological underestimates. In these instances, insoluble material precipitated from the digests after short storage at $4^{\circ}$ suggesting that aggregates were re-forming.

On raising the sodium chloride concentration from 0.15 to $2 \mathrm{M}$ in the agar medium (see Methods) aggregates were dispersed and did not reform. At this high salt concentration antigen-antibody interaction is confined to hydrophobic bonds, reducing the sensitivity of the immunological assay. However, by adjustment of the dilutions of standard and test plasmas, it was found that immunoreactive fibrinogen values again approximated closely to the expected value as compared with clottable and immunoreactive fibrinogen values of matched normal plasmas.

\section{Discussion}

From these results it will be evident that comparison of immunoreactive and clottable fibrinogen values yields information indirectly, but simply, about the functional utilization of fibrinogen. First, the measurements confirm the marked responsiveness of plasma fibrinogen levels to metabolic stresses. More elaborate studies of fibrinogen turnover, using radioisotopic tracers, have established that changes in the plasma concentration reflect alterations in the synthesis rate (cf, Regoeczi, Regoeczi, and McFarlane 1964; Blömback, Carlson, Franzén, and Zetterqvist, 1966).

In health, some variation has been attributed to diurnal 'random' fluctuation of fibrinogen metabolism (Reeve, Takeda, and Atencio, 1966). But in our hands, sequential studies in healthy males, who were presumably in a metabolic steady state in relation to synthesis and catabolism, have shown little change in level except in response to minor infections, etc. In healthy women, a wider range of fluctuations was encountered which was apparently related to the menstrual cycle. Studies on physiological variation in fibrinogen levels during pregnancy and childbirth will be reported separately.

Secondly, the increased concentration of fibrinogen found following surgery resembles that reported following injuries, eg, burns, as reported by Davies, Ricketts, and Bull (1966) and by Kukral, Zeineh, Dobryszycka, Pollitt, and Stone (1969), and shows similar time relations, suggesting an increased synthesis of fibrinogen as found following burns. In this respect, therefore, the response of fibrinogen, in resembling that of other 'acute phase reactant' serum proteins (cf Werner and Cohnen, 1969 ) in the postoperative period, is confirmed.

Additional information about the fate and behaviour of fibrinogen can be adduced by consideration of discrepancies between immunoreactive and clottable fibrinogen estimates. When the discrepancy takes the form of lower immunoreactive than clottable fibrinogen values, it has been found that this is associated with the presence of aggregates of the fibrinogen molecule in the plasma and with the occurrence of thrombosis. The characteristics of the aggregates resemble the "cryoprofibrin' shown by Shainoff and Page (1960) to be composed of heterogeneous fibrin intermediates formed as a result of thrombin activity in vivo. Their presence in the plasma of our patients within the first 24 to 48 hours of surgical intervention, ie, when thrombosis can be envisaged as occurring in the operative field, or in an individual with thrombosis in progress (see Fig. 5), is compatible with such an origin. It seems reasonable to assume that tissue damage at operation gives rise to massive release of thromboplastin and thus to considerable thrombin formation. Thrombin surplus to that utilized for local haemostasis and in excess of the amount capable of neutralization by plasma antithrombin would be considerably diluted in the circulation. At low concentration the enzyme might effect only partial and incomplete conversion of fibrinogen to 'cryoprofibrin' (see Fig. 1B).

The failure of fibrinogen in this abnormal physical form to give full quantitative immunodiffusion reactions may be due to one or more of the following: (1) the large size of the aggregates giving rise to very slow diffusion or actual precipitation in the agar; (2) an effective reduction in molar concentration of fibrinogen; or (3) aggregate-induced steric hindrance of access by antibody to reactive sites on the antigen. Whatever the mechanism, the occurrence of this anomaly appears to be an indication of thrombosis in progress in the body. From reference to Fig. 3 it can be seen that this anomaly may occasionally be encountered in healthy women in the menstrual phase, presumably then reflecting the occurrence of thrombosis in the deeper endometrial vessels. Apart from these circumstances, the anomaly has only been encountered in association with 'pathological' thrombosis of some magnitude. Our experience to date, for example, suggests that the anomaly is only detected in ischaemic heart disease when massive infarction, associated with intracardiac (mural) thrombosis due to thrombosis of a major coronary artery, occurs. While of limited value as a general 
screening test for coronary thrombosis, therefore, the detection of the anomaly may be of clinical significance in assessing other varieties of intra- or extravascular thrombosis.

Thirdly, when immunoreactive values are found markedly to exceed clottable fibrinogen values the discrepancy appears to be associated with active thrombolysis. The phenomenon appears to be a more sensitive indicator of the presence in the circulation of fibrin degradation products than the estimation of these products in serum by haemagglutination inhibition (Merskey et al, 1966) or other immunological methods of testing serum, presumably because these latter methods are also influenced by accelerated fibrinolysis in vitro due to increased output of plasminogen activator (Bennett, Ogston, and Ogston, 1967).

In comparing immunoreactive and clottable fibrinogen values statistically for normal subjects, the clottable value was taken to be the independent variable, free from error. However, our experience suggests that the occurrence of the discrepancy as a test for the elaboration of fibrin degradation products during thrombolysis may sometimes be obscured by spuriously elevated clottable fibrinogen values due to the occlusion of the degradation products in the clot. For example, underestimates of these products in serum in the presence of high levels in plasma (see Fig. 4) may arise for this reason. Other evidence in support of this conclusion, and the manner in which it can be demonstrated, will be published separately.

Despite this reservation, and without more elaborate measures to establish occlusion in clots, the occurrence of the anomaly of immunoreactive values in considerable excess of clottable fibrinogen values has nevertheless been demonstrable in about $70 \%$ of postoperative cases from about the third day onwards. If allowance is made for the occasional occurrence of the anomaly in the premenstrual phase, its presence otherwise is suggested to be an indicator of active thrombolysis following 'pathological' thrombosis.

Once again, in our hands the occurrence of this anomaly has proved to be more sensitive than immunoelectrophoresis of plasma as a means of detecting fibrin or fibrinogen breakdown.

We acknowledge gratefully the cooperation of the physicians and surgeons of the United Birmingham Hospitals in allowing us access to cases with sus- pected thrombosis or thrombolysis and, in particular to Professor J. Hardwicke for permission to present the results of a patient with renal vein thrombosis under his care.

\section{References}

Allison, A. C., and Humphrey, J. H. (1960). A theoretical and experimental analysis of double diffusion'precipitin reactions in gels, and its application to characterization of antigens. Immunology, 3, 95-106.

Bailey, K., and Bettelheim, F. R. (1955). The clotting of fibrinogen. I. The liberation of peptide material. Biochem. biophys. Acta (Amst.), 18, 495-503.

Bennett, N. B., Ogston, C. M., and Ogston, D. (1967). Studies on the blood fibrinolytic enzyme system following acute myocardial infarction. Clin. Sci., 32, 27-37.

Biggs, R., and Macfarlane, R. G. (1957). In Human Blood Coagulation and its Disorders, 2nd ed., p. 392. Blackwell, Oxford.

Blömback, B. (1967). In Blood Clotting Enzymology, edited by W. H. Seegers, p. 201. Academic Press, New York.

Blömback, B., Carlson, L. A., Franzén, S., and Zetterqvist, E. (1966). Turnover of ${ }^{131}$ I-labelled fibrinogen in man. Acta med. scand., 179, 557-574.

Davies, J. W. L., Ricketts, C. R., and Bull, J. P. (1966). Studies of plasma protein metabolism. III. Fibrinogen in burned patients. Clin. Sci., 30, 305-314

Farrell, G. W., and Wolf, P. (1970). A simple method of preparing monospecific anti-human fibrin serum in rabbits. J. med. Lab. Technol., 27, 312-316.

Farrell, G. W., and Wolf, P. (1971). Studies on thrombosis and thrombolysis. Med. lab. Technol., 28, 310-318, 319-327, 328-336.

Fearnley, C. R., and Chakrabarti, R. (1966). Fibrinolytic treatment of rheumatoid arthritis with phenformin plus ethyloestrenol. Lancet, 2, 757-760.

Kukral, J. C., Zeineh, R., Dobryszycka, W., Pollitt, J., and Stone, N. (1969). Metabolism of plasma proteins in injury states. I. Turnover rates of fibrinogen in burned patients labelled with $\left({ }^{35} \mathrm{~S}\right)$ methionine. Clin. Sci., 36, 221-230.

Lipinski, B., Wegrzynowicz, Z., Budzynski, A. Z., Kopeć, M., Latallo, Z. S., and Kowalski, E. (1967). Soluble unclottable complexes formed in the presence of fibrinogen degradation products (FDP) during the fibrinogen-fibrin conversion and their potential significance in pathology. Thrombos. diathes. Haemorrh. (Stuttg.), 17, 65-77.

McFarlane, A. S., Todd, D., and Cromwell, S. (1966). Fibrinogen catabolism in humans. Clin. Sci., 26, 415-420.

Merskey, C., Kleiner, G. J., and Johnson, A. S. (1966). Quantitative estimation of split products of fibrinogen in human serum, relation to diagnosis and treatment. Blood, 28, 1-18.

Nussenzweig, V., Seligmann, M., and Grabar, P. (1961). Les produits de dégredation du fibrinogène humain par la plasmine. II. Etude immunologique: mise en évidence d'anticorps antifibrinogène natif possédant des specificités différentes. Ann. Inst. Pasteur, 100, 490-508.

Reeve, E. B., Takeda, Y., and Atencio, A. C. (1966). Some observations on the mammalian fibrinogen system in non-steady and steady states. Protides biol. Fluids, 14, 283-294.

Regoeczi, E., Regoeczi, G. E., and McFarlane, A. S. (1964). Relation between rate of catabolism, plasma concentration and pool size of fibrinogen. Pflügers Arch. ges. Physiol., 279, 17-25.

Shainoff, J. R., and Page, I. H. (1960). Significance of cryoprofibrin in fibrinogen-fibrin conversion. J. exp. Med. 116, 687-707.

Takeda, Y. (1966). Studies of the metabolism and distribution of fibrinogen in healthy men with autologous ${ }^{125} \mathrm{I}$-labeled fibrinogen. J. clin. Invest., 45, 103-111.

Werner, M., and Cohnen, G. (1969). Changes in serum proteins in the immediate postoperative period. Clin. Sci., 36, 173-184.

Wolf, P., and Walton, K. W. (1965). Investigation of a quantitative anomaly encountered in the assay of fibrinogen by immunodiffusion. Immunology, 8, 6-24. 